\title{
BIOLOGICAL CONTROL AND IMPACTS ON NON-TARGET SPECIES
}

\author{
J.M. CULLEN \\ CSIRO Entomology, GPO Box 1700, Canberra, ACT 2601, Australia
}

\begin{abstract}
Several examples are known of utilisation of non-target native species by biological control agents for invertebrate pests and weeds, but analyses of the consequences for the species are lacking. Four scenarios are considered, depending on the extent of co-occurrence of agent and nontarget and the agent's relative preference for non-target and target species. Together with examination of some field examples, this suggests that in most cases impacts are limited, but the potential exists for serious impacts. A risk analysis approach is advocated to proposals for release with some examples. Lack of relevant research data is a major problem.

Keywords: Biological control, non-targets, host-specificity, risk assessment
\end{abstract}

\section{INTRODUCTION}

The environmental credentials of classical biological control as a safe, specific alternative to chemical control of pests and weeds have been severely questioned in recent years (Howarth 1991) resulting in considerable and sometimes animated debate. With the increase in general concern over loss of biodiversity has come the concern that attack by an introduced biological control agent on species other than the target pest or weed, particularly if native, could be causing biodiversity loss. The irreversibility of successful classical biological control introductions is highlighting consideration of the consequences of such effects (Samways 1994).

The introduction of vertebrates as biological control agents has been a very blunt and non-specific instrument, often with serious deleterious side effects and is no longer considered as a serious option (McEvoy 1995). This paper is concerned only with the introduction of agents, invertebrates and fungi, for the control of invertebrate pests and weeds. While most emphasis is placed on the importance of habitats for conservation, more attention will be paid to species in this case as that is the primary level at which effects of individual introductions might be registered. I will attempt to examine scales of possible impact, the factors that might influence that impact and finally make some observations on how we are trying to manage the process of introductions.

\section{LEVELS OF IMPACT}

Andres (1981), Diehl and McEvoy (1990), Harris (1988) and Turner (1985) have listed several North American cases of agents imported for weed biological control. In Australia, it has been known for some time that the parasiteTrissolcus basalisimported to Australia from several sources for biological control of green vegetable bug (Nezara viridula), utilises several Australian pentatomid species (Waterhouse and Norris 1987). In New Zealand, Green (1984) recorded the introduced parasite Trigonospila brevifacies attacking several native Lepidoptera while Barrattet al.(1997) have recently documented the parasitism of several native weevil species by the braconidMicroctonus aethiopoides released for control of Sitona discoideus. There is no doubt that the phenomenon occurs, but there is considerable doubt over its significance. However, it is not sufficient to claim there is no evidence of any significant effect because in the vast majority of cases, nobody has looked. What can be done is to review some of the theoretical possibilities and consider them in the light of what little information is available from the field. Four scenarios are examined below, which parallel in a more general sense those suggested

Proc. 50th N.Z. Plant Protection Conf. 1997: 195-201 
by Hopper (1995) in his analysis of possible impact on endangered arthropod species in the US.

1. When a parasite is established on a target host, or a phytophagous insect on a weed, for its possible control, if it is completely monophagous there are no direct non-target effects. There may be some indirect effects, for instance if the target has become a significant part of a food chain. Whether the target is exotic or native, the critical decision is whether it should be controlled in the first place, given that it has some benefit. This is a question to be resolved elsewhere. Some suitable mechanisms are in place, usually for weeds, but not for arthropods. In 1990 a workshop was held in Australia to discuss procedures for biological control introductions in relation to quarantine and environmental concerns (Cullen 1991). The only recommendation not accepted was to examine the case for an approval system for arthropod targets for biological control, in addition to weeds. While this remains an issue, particularly for native pests, it is not central to the present debate and will not be pursued further.

2. If a parasite or predator for an invertebrate pest or a weed herbivore can utilise one or more species in addition to its normal host, but never comes into contact with them due to temporal or spatial separation, there will be no impact.

3. If a parasite or weed herbivore also utilises one or more co-occurring species in addition to its normal host, but to a lesser extent, some non-target impacts may be possible. Such a situation is probably fairly common in arthropod biological control, but less so in weeds, though Turner (1985) lists 7 (21\%) examples of the 33 agents introduced for weed biological control in North America. The population level of the agent is determined primarily by the target host. Thus at high target host density the biological control agent could also be at high levels and there could be spill over effects on non-target species. However, if numbers on the target host are high, it is likely that the target host will decline and the effect should be transitory. Minor damage was reported on some plants at the peak of the prickly pear program, but has not been seen since (Waterhouse and Norris 1987). The long term effect concerns the significance of an additional small level of mortality on the non-target species. In many cases it may substitute for an alternative mortality factor with negligible difference to the total mortality. For an arthropod for instance, mortality from general predators will often be determined by the commonness of the species. If it is slightly less common, it is likely to be preyed on less and the sum effect may not change. In the case of a plant, if the stage affected, e.g. seed production, is succeeded by a period of intraspecific competition, e.g. seedling survival, the overall effect may be minimal.

However, it is possible to envisage situations where a small level of mortality may have a more significant effect. It is likely that there are habitats towards the margin of a species' range where survival is marginal and additional mortality could swing the balance towards extinction in that habitat. If the agent needs the target host present to be able to maintain its numbers, then the area of co-occurrence of target, agent and non-target would need to include such marginal areas. Also, in such habitats, fluctuation of the non-target from season to season, perhaps dependent on climate or food resource, would be common and perhaps often of a scale to blanket any additional small mortality. If a species is traditionally rare in such areas, and in the more general case, rare or endangered over the whole of its range, it would also be less likely to be found and subject to attack. Therefore, to be significant in the conservation of a species, additional mortality from an introduced biological control agent would have to be additive rather than substitutive, it would have to occur in those habitats where the non-target was at risk, it would have to remain significant when the non-target species was difficult to find and its effects should not be overridden by other sources of fluctuation. Such a combination of conditions is probably rare, though good data is scarce.

4. If an introduced biological control agent attacks one or more non-target species as commonly or more commonly than the target host, impacts can clearly be more significant. In such circumstances, the population of the agent is no longer determined principally by the target species, but rather by a complex, of which the target is only one member. High numbers may well be maintained on one or more species to the 
detriment of any individual species, particularly if it is a preferred host. If this is the target, control may be extremely effective. If it is not, a native non-target species may suffer considerable impact.

Debates as to whether target species, e.g.Levuana iridescens, or non-target species, e.g. Heteropan dolens, have been rendered extinct by biological control agents are to some extent irrelevant. As pointed out by Lawton (1986) extinction is certainly a possibility over part or the whole of a range. In reality, whether or not impact is severe enough to cause extinction or even significant depression, will depend on several factors to do with variation in populations of all possible hosts in space and time and the distribution of the agent by region and habitat.

\section{FIELD EXAMPLES}

Unfortunately there is little field data available to improve our understanding of the critical scenarios 3 and 4 above and the distinction between them. The observations of Turner et al. (1987) on Rhinocyllus conicus attacking native Cirsium spp. showed the number of adult $R$. conicus obtained from 100 heads of different species ranging from 1 to over 1000 . The rarer species were at the low end of the range. The higher figures, from the commoner Cirsium species, are as high as found on target species and enough to suggest $R$. conicus can maintain itself on these species and seriously depress seed production, though without a knowledge of seed dynamics it cannot be determined whether there is significant population depression.

Diehl and McEvoy (1990) document quite significant utilisation of Senecio triangularis by Tyria jacobaeae, whose target host is Senecio jacobaea, but with considerable variation between habitats. Depending on the relative importance of reproduction by seed and rhizomes, there could be impact on roadside stands, but not in meadows.

In Australia,Trissolcus basalis is certainly able to maintain itself on eggs of Oechalia schellenbergii, Cermatulus nasalis andAgonoscelis rutilis, with high levels of parasitism often observed. These species are still common and there is no evidence of decline (Sands 1997), but accurate data is lacking.

Nafus (1993) provides an example where parasitism of two non-target species of butterflies by an introduced wasp is effectively swamped by normal predation by native ants, though the author expresses some concern over impact when predation is low.

The parasitism of some native New Zealand weevils, particularlyIrenimus aemulator, by Microctonus aethiopoides, both in laboratory tests and in the field, is as high or higher than on the target host Sitona discoideus (Barratt et al. 1997), suggesting that there is the possibility of serious impact. It will be important to know in time whether this is in fact the case, and if not, why not.

Overall, the small amount of information available is not inconsistent with the view that situations characterised by scenario 3, i.e. less common attack on a non-target species, rarely cause a problem and that while scenario 4 could include examples resulting in severe impact, they need not do so, depending on other factors. McEvoy (1996) suggests that non-target effects of weed biological control agents tend to be localised in space and time, but the probability and consequences of attack on non-target plants need to be estimated before safety concerns can be put to rest. To do this properly, for arthropod or weed biological control, better data is desperately required. A few well studied case histories would be immensely valuable.

\section{VARIATION IN THE LEVEL OF UTILISATION OF TARGETS AND NON TARGETS}

The relative level of attack between target and non-target species is clearly a basic input to analysing the probability of impact, but is not a constant. Apart from taxonomic relationship to the target, a number of factors have been demonstrated to influence host selection and hence relative preference, including habitat (Vinson 1985), host shape and size (Salt 1958, Gross 1993), egg load and encounter rates on targets and non-targets (Janssen 1989). Of these, habitat is particularly important, including, the host plant or part of the host plant (Price et al. 1980), nature of the surrounding vegetation (Root 
1973) and possibly location, e.g. open grassland cv shaded rainforest. Evidence for the latter is not clear, though many insects are known to seek or avoid shade, including weed herbivores. Better evidence of its influence on parasites, independent of presence or absence of hosts, would be valuable.

A generality from the above and from Lawton's (1986) analysis of the impact of polyphagous parasites on host communities is that effects on non-target species will be more probable when the species have similar niches to the target and will decrease with increasing niche distance.

\section{DISCUSSION}

A large number of factors obviously influence the extent of utilisation of non-target species and the impact this might have. Importantly, as well as negligible impact, the range of possible outcomes does include significant population decrease and possible extinction.

It could be suggested that because of this possibility, biological control should be halted. This is not a tenable solution. The environmental harm that may be caused by other approaches to pest and weed management is considerable and some problems, themselves a major disaster for biodiversity, e.g. Mimosa pigra in northern Australia, cannot be effectively tackled without biological control as part of their management.

The issue becomes one of risk analysis, assessable by better defining the probabilities. If the overall probability of impact for conservation of non-target species is low, biological control is still a favoured option, both environmentally and economically. If the probability of impact is high, then a judgement will need to be made, balancing the risks and benefits with as predictive an analysis of both as possible. This should not be a judgement by the biological control practitioner, but by an informed and independent review process. Statements that a certain outcome may happen are not particularly helpful without an assessment of the probability of it happening. Nor are sweeping generalisations useful when individual cases need to be assessed on the relevant information available, each situation reflecting a unique set of potential interactions between control agent, target and non-target (McEvoy 1996).

In our current state of knowledge, how feasible is such an approach? Sands (1997) has pointed out some of the difficulties, but in fact, judgements are already being made on this basis. I would like to consider three recent examples from Australian programs.

1. In laboratory tests, the rust Uromyces heliotropii, potential control agent for Heliotropium europaeum, was found to produce low level infection on young plants (6-9 weeks old) of the Australian native species Heliotropium crispatum. Repeated reinfection of $H$. crispatum, using spores from this species, showed attenuated infection after five generations, but concern was expressed over possible infection of this species in the field. A number of factors were considered:

(a) Geographical and temporal separation. H. crispatum is confined to the northwest of Australia where it is a winter growing annual.H. europaeum is a summer growing annual in southern Australia, the nearest area being south-western Australia, at least $600 \mathrm{~km}$ away.

(b) Dispersal. When $H$. crispatum is available for infection, the only spores available are non-dispersing, overwintering spores in ground litter and the winds between northern and southern Western Australia are predominantly west-east.

(c) Probability of establishment. An analysis of overnight temperatures and dew points in relation to infection requirements showed that conditions for infection were probably never achieved in the inland distribution, and infrequently in the coastal distribution, of $H$. crispatum.

(d) Density. H. crispatum occurs as scattered individual plants compared with the dense stands of $H$. europaeum.

Overall, the probability of infection of $H$. crispatum from any infection established on H. europaeum was considered extremely low, while the possible consequence of any such infection, i.e. impact, was also low. The risk of damage toH. crispatum was considered negligible (Hasan and Delfosse 1995).

2. In the second case, the eriophyid mite Aculus hyperici was approved for release to 
control Hypericum perforatum in the knowledge that it could maintain itself for several generations in quarantine on the Australian native species Hypericum gramineum. The distribution of $H$. gramineum overlaps significantly with that of $H$. perforatum, but includes many areas where $H$. perforatum is absent. Factors taken into account were:-

(a) Populations of A. hyperici progressively declined over time on H. gramineum from an initial high level established by inoculation. It was considered unlikely to be able to maintain populations on $H$. gramineum in the absence of $H$. perforatum.

(b) Damage is slow to develop on host plants and dependent on maintaining high populations of $A$. hyperici.

(c) Populations of $A$. hyperici could only be maintained by colonisation from neighbouring $H$. perforatum while its density was high and populations of $A$. hyperici also high. In such a case, $H$. perforatum was expected to decline and any effect on $H$. gramineum would be transitory.

In this case, the probability of infection was probably initially medium-high, but low subsequently, while the potential impact was medium.

The other factor in this case was that $H$. perforatum was itself a threat to conservation areas and other methods of control were not available for such environments (CSIRO 1991).

3. The third case concerns consideration of the tachinid Trichopoda giacomellii for control ofNezara viridula (Sands and Coombs 1996). This parasite was exposed to 12 potential non-target species, ten Pentatomidae and two Coreidae. In cage tests, significant oviposition was obtained on six species, three to a level equal to or exceeding the level on $N$. viridula. However, the other three were unable to support development of $T$. giacomellii. Discussion centred on:-

(a) Fecundity and longevity of the parasite was lower in females emerging from the three non-target species, suggesting they were sub-optimal for development.

(b) Two of the three species, while occurring in similar habitats to $N$. viridula, were common and have pest status themselves. They also occur elsewhere away from N. viridula, e.g rainforests.

(c) The third species is principally or wholly a species of rainforest habitats. $N$. viridula is a species of open cropping systems and observations over a long period in South America, the origin ofT. giacomellii, established it was a species of crop and grasslands and not found in rainforests.

Depending on the species of non-target and its habitat, the probability of utilisation was medium, with low impact, or low probability of utilisation with medium impact.

Each of these assessments was made on the basis of laboratory tests and other relevant knowledge of the ecosystems in which the agents would operate. The critical requirement now is to follow up the predictions with field assessments to test their validity. So far, this has been pursued for Aculus hyperici with the conclusion that the predictions were partially correct, but that further field monitoring was required (Willis 1994). The link between data obtainable in the laboratory and the situation in the field is critical in this process and needs to be better understood. Relevant studies are rare and the work of Barratt et al. (1995) is therefore particularly valuable in trying to test the predictability of laboratory tests that could form the basis for future protocols in New Zealand.

In one of the examples described above, accurate observation from the country of origin of the agent, was very useful. Observation and experimental manipulation has been used to advantage in other proposals (Clement and Cristofaro 1995).

No attention has been given in this account to indirect effects, e.g. competitive displacement (McEvoy 1996), nor to genetic change and host shifts. These are even more speculative risks with infinite possibilities, though Lawton (1985) heavily discounts the risk of host shifts. While we need to be aware of these possibilities and act if information is available, there is little capacity to put meaningful probabilities on these risks at present. 


\section{CONCLUSION}

Biological control has to continue as one of the most valuable components of sustainable production and environmental management, but can only do so as it takes account of a broader range of possible risk. To do this more effectively, research data is required in some key areas, e.g.

- The conservation consequences of differing degrees of additional mortality in a system; case studies of known utilisation of non-target species are urgently required.

- The extent to which biological control agents are limited to particular ecosystems independent of the occurrence of possible hosts;

- The reliability of predictions from laboratory tests to field situations regarding levels of attack on non-targets;

- The extent to which manipulation of hosts and habitats in the region of origin of a biological control agent can yield important information.

We should proceed with as good an assessment of the probability of future events, both beneficial and detrimental, as possible. Mistakes will possibly be made, as they will by those charged with the specific responsibility of conservation, because of the lack of detailed knowledge. Decisions have to be made in real time, not when we are omniscient. We can try to make as few of them as possible.

\section{REFERENCES}

Andres, L.A., 1981. Conflicting interests and the biological control of weeds. Pp. 11-20 In: Proc. V Int. Symp. Biol. Contr. Weeds, 22-27 July 1980, Brisbane, Australia. E.S.Delfosse (Ed) CSIRO, Melbourne.

Barratt, B.I.P., Evans, A.A., Ferguson, C.M., Barker, G.M., McNeill, M.R. and Phillips, C.B., 1997. Laboratory nontarget host range of the introduced parasitoidsMicroctonus aethiopoides and Microctonus hyperodae (Hymenoptera: Braconidae) compared with field parasitism in New Zealand. Env. Entomol: (in press).

Clement, S.L. and Cristofaro, M., 1995. Open-field tests in host-specificity determination of insects for biological control of weeds. Biocont. Sci. Tech. 5: 395-406.

CSIRO Division of Entomology. 1991. The host-specificity of Aculus hyperici (Liro) (Acarina: Eriophyidae) in relation to different species in the genus Hypericum. Unpublished report to Australian Quarantine and Inspection Service. $15 \mathrm{pp}$.

Cullen, J.M., (Ed) 1991. Proceedings of a Workshop on Environmental Conservation and Biological Control, unpublished report to Standing Committee on Agriculture and Council of Nature Conservation Ministers.

Diehl, J. and McEvoy, P.B., 1990. Impact of the cinnabar moth (Tyria jacobaeae) on Senecio triangularis, a non-target native plant in Oregon. Pp. 119-26.In: Proc. VII Int. Symp. Biol. Contr. Weeds, 6-11 Mar 1988, Rome, Italy. E.S.Delfosse (Ed) Min.Agric.For., Rome and CSIRO, Melbourne, Australia,

Green, O.R., 1984. New Zealand host and locality records for an introduced tortricid parasite Trigonospila brevifacies (Diptera: Tachinidae).New Zealand Entomol. 8: 69-71.

Gross, P., 1993. Insect behavioural and morphological defences against parasitoids.Ann. Rev. Ent. 38: 251-73.

Harris, P., 1988. Environmental impact of weed biocontrol insects. Biosci. 38: 542-8.

Hasan, S. and Delfosse, E.S., 1995. Susceptibility of the Australian native,Heliotropium crispatum, to the rust fungus Uromyces heliotropii introduced to control common Heliotrope, Heliotropium europaeum. Biocontr. Sci. Tech. 5: 165-74.

Hopper, K.R., 1995. Potential impacts on threatened and endangered insect species in the continental United States from introductions of parasitic Hymenoptera for the control of insect pests. Pp. 64-74. In: Biological Control: Benefits and Risks, H. Hokkanen and J.M.Lynch (Eds); Cambridge University Press, Cambridge.

Howarth, F.G., 1991. Environmental impacts of classical biological control.Ann. Rev. Ent. 36: 485-509.

Janssen, A., 1989. Optimal host selection by Drosophila parasitoids in the field.Funct. Ecol. 3: 469-79.

Lawton, J.H., 1985. Ecological theory and choice of biological control agents. Pp. 13- 
26. In: Proc VI Int. Symp. Biol. Contr. Weeds, 19-25 Aug 1984, Vancouver, Canada, E.S.Delfosse (Ed). Agric. Canada.

Lawton, J.H., 1986. The effect of parasitoids on phytophagous insect communities. Pp. 265-287. In: Insect Parasitoids. J.K.Waage and D.J.Greathead (Eds). Academic Press, Orlando,Fl.

McEvoy, P.B., 1995. Testing Biocontrol Agents and Microbial Biopesticides for Host Specificity, unpublished contractor report for Office of Technology Assessment, U.S. Congress, Washington.

McEvoy, P.B., 1996. Host specificity and biological pest control. Bioscience 46: 401405.

Nafus, D.M., 1993. Movement of introduced biological control agents onto nontarget butterflies, Hypolimnas spp. (Lepidoptera: Nymphalidae). Env. Entomol. 22: 26572.

Price, P.W., Bouton, C.E., Gross, P., McPherson, B.A., Thompson, J.N. and Weiss, A.E., 1980. Interactions among three trophic levels: influence of plants on interactions between insect herbivores and natural enemies. Ann. Rev. Ecol. Syst. 11: 41-65.

Root, R.B., 1973. Organisation of plant-arthropod associations in simple and diverse habitats: The fauna of collards (Brassica oleracea). Ecol. Monogr. 43: 95-124.

Salt, G., 1958. Parasite behaviour and the control of insect pests. Endeavour 17:145148.

Samways, M.J., 1994. Insect Conservation Biology, Chapman and Hall, New York. 358 pp.

Sands, D.P.A., 1997. The safety of biological control agents: assessing their impact on beneficial and other non-target hosts. Mem. Mus. Victoria 56: 611-616.

Sands, D.P.A. and Coombs, M.T., 1995. Application to releaseTrichopoda giacomellii (Blanchard) (Diptera: Tachinidae), a biological control agent for green vegetable bug, Nezara viridula (L.) (Hemiptera: Pentatomidae). Unpublished report to Australian Quarantine and Inspection Service, 17pp.

Turner, C.E., 1985. Conflicting interests and biological control of weeds. Pp. 203-25.In: Proc VI Int. Symp. Biol. Contr. Weeds, 19-25 Aug 1984, Vancouver, Canada, E.S.Delfosse (Ed). Agric. Canada.

Turner, C.E., Pemberton, R.W. and Rosenthal, S.S., 1987. Host utilisation of native Cirsium thistles (Asteraceae) by the introduced weevil Rhinocyllus conicus (Coleoptera: Curculionidae) in California. Env. Entomol. 16: 111-115.

Vinson, S.B., 1985. The behaviour of parasitoids. Pp. 417-69In: Comprehensive Insect Physiology, Biochemistry and Pharmacology, G.A.Kerkut and L.I.Gilbert (Eds); Pergamon Press, New York.

Waterhouse, D.F. and Norris, K.R., 1987. Biological Control: Pacific Prospects. Inkata Press, Melbourne. 454 pp.

Willis, A.J., 1994. The ecology of Hypericum gramineum with reference to biological control of $H$. perforatum by the mite, Aculus hyperici. Unpublished Ph.D. thesis, Australian National University, Canberra. 